of hesitation that we instituted this measure, for we were familiar with the fact that mere starvation could be the cause of a very rapid increase of abnormal acids. Perhaps, somewhat contrary to our expectations, we have often noted a remarkably prompt improvement to follow on a period when nothing but water and a solution of sodium bicarbonate was given. We have seen the stupor disappear and respirations fall almost to normal over night with this simple treatment. We do not consider that this improvement was entirely due to the sodium bicarbonate, for under other conditions of feeding recovery, if it took place at all, was much slower.

A very important part of our treatment has been a thorough cleaning out of the bowels and especially of the small intestines. High colon irrigations and stomach washing did not accomplish much improvement if our efforts ceased at this point. Nearly always such a catharsis resulted in a very nasty, foul smelling stool

The use of sodium bicarbonate is to be recommended as a valuable temporary measure. It has much the same relation to acidosis that the cold pack has to temperature. It serves to keep the patient alive until other measures which require time become effective. It should be pushed until the reaction of the urine is alkaline or the blood shows a normal reaction. We have made it a practice to give the sodium bicarbonate in every possible way - by stomach, by the colon, subcutaneously and intravenously - as the urgency of the symptoms seemed to dictate.

As we have tentatively connected attacks of acidosis with the milk - and perhaps most frequently with milk which contains an unduly high bacterial count a very essential part of our treatment has been the elimination of milk from the diet for a considerable period of time. Our most successful cases have been those in which we have made a very slow return to the proteins. To an extent we have made vegetable proteins take the place of animal proteins; accordingly these children have been given thick gruels which were cooked for many hours. With the addition of sugar as the symptoms seem to warrant, the caloric requirements for a time, at least, can be met.

The mortality rate among children suffering from an attack of acidosis is so great as to make it a condition which should demand not only our interest but also our earnest investigation into its cause and treatment. We have suggested the close relation of acidosis to the milk supply and especially to the decomposition products of the proteins of milk. We have outlined a method of treatment which is more satisfactory than any that we have previously employed, and which is based on the assumption of the close relationship of milk and this type of acidosis.

Ocular Reflex of Auditory Origin.-The French authorities have designated as the standard of hearing for recruits that they can hear a whisper at 0.5 meter and the speaking voice at 4 meters. Simulation of deafness to escape military service is occasionally encountered, and it can be unmasked by the behavior of the cyes when a sudden, unexpected sound strikes the ear from nearby. With a sudden start from the loud sound close to the ear the subject winks, and the pupil may contract. This brief defensive closure of the eyelids of course does not occur if the subject fails to hear the sound. Molinie says in his description of the simple technic for the tests (Revue de laryngologie, 1916, p. 385) that it automatically sifts out the really deaf who might be accused of malingering otherwise.

\section{MYASTHENIA GRAVIS, WITH THYMOMA *}

\section{W. A. JONES, M.D. MINNEAPOLIS}

The patient whose case history is here given presented himself at the City Hospital in Minneapolis in November, 1915. The first and cursory examination suggested the usual type of bulbar paralysis, but after the patient had been admitted to the permanent ward and studied more carefully, the diagnosis was changed to that of myasthenia gravis.

The family history was as follows: The mother was living and well at 89 ; the father died at 60 , the cause of death not known; two brothers were living and well; one brother died of pneumonia; there were no sisters; the wife died at 38 , cause of death not known; there were two children, both of whom were living and well.

The history of the antecedents, so far as the patient was able to remember, revealed no nervous or mental trouble in the family, on either the paternal or the maternal sicle. There was no history similar to the condition from which the patient suffered in any member of the family.

The personal history showed that the patient had always been a strong, healthy man, and had never been seriously ill prior to the onset of his present trouble. He had the usual exanthemata of childhood, from which he made good recoveries without sequelae. Twenty-seven years before he had an attack of malaria, but was able to be about, and was at no time seriously ill. He had no other history of illness. He had suffered no serious injuries of any kind, and never had been operated on. His habits had always been moderate. He used very little coffee, no tea, and no alcohol, but had been for some time a heavy user of tobacco, and gave a history of more or less persistent constipation. Prior to the attack his appetite had been good, and he had slept well. $\mathrm{He}$ denied any venereal trouble, nor could any be elicited from laboratory findings. He had an inguinal hernia, but it was not troublesome or annoying. He had occasional attacks of tonsillitis, and he had hemorrhoids.

To the best of the patient's knowledge, he was in perfectly good health three years before. About May, 1912, following an exposure to cold while working in water as a ship's carpenter, he noticed within a few days a marked weakness of his neck muscles, and within two weeks he had developed a condition similar to the one from which he suffered when examined. The symptoms came on rather slowly, and were associated with slight pain and soreness in the muscles of the shoulder and the back of the neck, followed by a general muscular weakness, and particularly by an inability to close his mouth, to swallow solid foods, or to drink liquids. This condition progressed until the patient became very emaciated, and was almost unable to take nourishment at all. This illness lasted a few weeks, when he began to improve and recovered his strength; and within six months after the onset of his primary symptoms, he claimed that he was perfectly well, and during the interim he did not recall that he had felt any weakness of muscles or anv inability to swallow or to speak.

Up to February, 1915, he was in his usual good health, and was able to attend to his work. During the month of February, 1915, without apparent catuse, unless it was a slight cold, as he expressed it, following exposure, the abovenamed symptoms began to return. There was pain in the jaw, but not of a severe type. Gradually the muscles of the neck and throat became weak, and he was unable to move his jaw or to swallow or drink with his usual ease. At the time of his admission into the hospital in November, 1915, he had a slight ptosis of the right eyelid. The weakness that he complained of was most marked in his neck muscles, but the entire musculature was more or less involved, and during the time that he was under observation he was

* Read before the Section on Nervous and Mental Diseases at the Sixty-Seventh Annual Session of the American Medical Association Detroit, June, 1916 
unable to walk, to make any marked exertion, or to eat a full meal. After a good night's rest the patient was very much better, but as the day went on his weakness became more marked. At the beginning of a meal swallowing and other movements of the jaw were comparatively free; but after a few moments the muscles tired, and he was unable to take further nourishment. It was difficult for him to blow his nose, and he was otliged to have his mouth closed and his chin held in position until he could force air through the nostrils. After talking for a few moments he was obliged to hold his lower jaw with the aid of his hand. Aside from this general weakness, and particularly of the muscles of the neck and jaw, and his inability to finish his meal, the patient felt fairly comfortable.

Neurologic examination seemed to show the mental condition to be normal for a man of his age. His speech was rather indistinct and slow, rather characteristic of his muscular weakness.

The first cranial nerve was normal. The second showed that vision was fair in both eyes and he had normal eyegrounds.

The ocular muscles, controlled by the third, fourth, and sixth nerves, were normal, with the exception that the right rectus was somewhat lax. He had no double vision and no nystagmus. The pupils, however, were irregular and slightly unequal, the right being larger than the left; both reacted sluggishly to light, but were normal for accommodation.

With the exception of a slight ptosis of the right eyelid, and the impairment of the external rectus muscle on the right side, there was nothing further to implicate the third and sixth nerves.

There was an apparent atrophy in both masseter muscles, and a marked inability to move the lower jaw. As a result of this involvement of the fifth nerve the mouth was open most of the time.

The seventh nerve presents no irregularities.

The eighth nerve showed that there was a moderate amount of deafness in the left side.

The tongue and palate were apparently normal. There was no facial or tongue tremor, except as was shown by his inability to swallow, and the fact that his speaking voice was below par, and any continued effort on his part to talk was accompanied by rapid muscular tire.

There was apparently no sensory disturbance to cotton, pinpoint, deep and superficial muscle sense, or vibration sense.

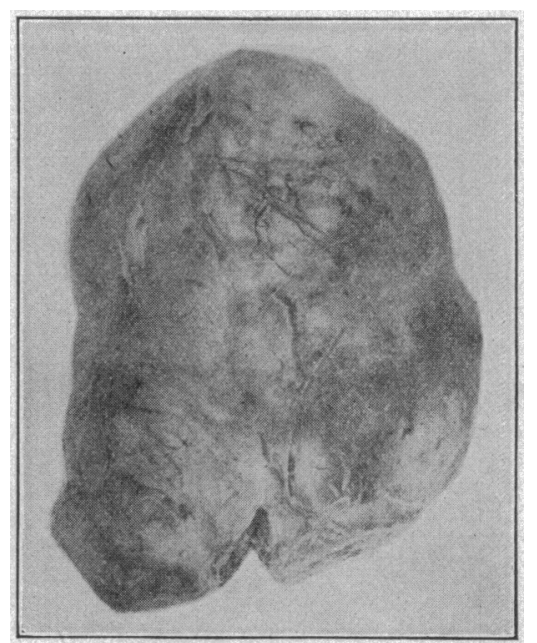

Fig. 1, - Photograph, natural size, of thymic region surrounded by firm capsule, with no perforations. tumor removed at necropsy, situated in

The biceps reflex was present and also the triceps. The supinator was active, and the patellar, active plus. The Achilles and abdominal and cremasteric were not obtained.

Coordination as far as was determinable was unimpaired.

The blood count was practically normal. A laboratory investigation of blood and spinal fluid showed the Wassermann negative. The urinary findings were also negative, eliminating any probable disease of the kidneys.

During the patient's entire stay at the hospital there was but little change from week to week in his condition. At times he felt better and stronger, and was able to move about in bed better at times; but there was no evidence of any marked change, with the exception of his dyspnea. On Dec. 10, 1915, he complained in the afternoon of a general fulness of his chest, an evident dyspnea. A hypodermic of $1 / 200$ grain of atropin was given, with the hope that it might relieve his distress. Ten minutes after the hypodermic was given he died suddenly.

A necropsy was made on Dec. 11, 1915, twenty-four hours after death, the report of which is as follows:

The body is well developed and fairly well nourished; pupils, $5 \mathrm{~mm}$. in diameter and equal, no edema, no special marks.
The peritoneal cavity contains no excess fluid and no adhesions. Intestinal surfaces are smooth and glistening. Appendix is $8 \mathrm{~cm}$. in length and free. Diaphragm extends to the fifth rib on either side. Mesenteric lymph nodes are firm and slightly enlarged.

There are no adhesions and no excess fluid in the pleural cavities.

The pericardial cavity contains about 3 c.c. of thin, clear fluid.

The heart weighs $275 \mathrm{gm}$. The epicardium is smooth and clear. The mitral valve shows a slight diffuse thickening. There are no lesions in the other valves. The endocardium is smooth and clear. The muscle is pale and firm. The root of the aorta shows no lesions.

The left lung shows $a *$ firm, whitish thickening of the pleura at the apex; thickness, about $6 \mathrm{~mm}$.; dimensions of area, 2 by $1 \mathrm{~cm}$. The lungs crepitate throughout. They are very deeply colored with black pigment. Throughout the lungs is a considerable number of small miliary nodules of firm consistence. In the apexes the miliary nodules show firm fibrous tissue of a black color. The cut surfaces of the lungs are fairly dry. The right lung weighs $375 \mathrm{gm}$., left $362 \mathrm{gm}$. There is an old healed apical tuberculosis of the lungs; old healed miliary tuberculosis of lungs.

The spleen weighs $130 \mathrm{gm}$. The capsule is wrinkled and slightly thickened. The pulp is fairly firm. The cut surface shows prominent lymph nodes.

The liver weighs $1,300 \mathrm{gm}$. The capsule is smooth and glistening. One small miliary nodule is noted on the anterior surface, resembling in structure those described in the lung. The cut surface shows no special markings. Lymphorrhages are numerous in the liver, and a few were found in the suprarenals.

The pancreas, gastro-intestinal tract and suprarenals show no gross lesions.

The left kidney weighs $108 \mathrm{gm}$; the right, $112 \mathrm{gm}$. They are of a dark red color. The capsules strip readily, exposing smooth surfaces. The cortices are about $7 \mathrm{~mm}$. in thickness and of a dark red color.

The bladder and genitalia show no pathologic condition.

The aorta is fairly elastic. A few very small, elevated, yellowish areas are noted.

A tumor is found in the thymic region, just above the heart and immediately behind the sternum. It is embedded in loose connective tissue, and is not adherent to the sternum or to any of the thoracic viscera. Microscopic examination of the adipose tissue surrounding the tumor shows the usual senile thymic tissue. The tumor is easily shelled out of the connective tissue envelope. It weighs $60 \mathrm{gm}$., is $6 \mathrm{~cm}$. long, $3.5 \mathrm{~cm}$. deep anteroposteriorly, and about $4 \mathrm{~cm}$. in transverse direction. It is completely enclosed in a strong fibrous capsule about $0.5 \mathrm{~mm}$. thick, which is not perforated at any point. The cut surface is of soft consistence, somewhat softer than the thymus of a child, and is studded with hemorrhagic areas. The cut surface is of a light gray color except in the portions containing blood. No lobules can be seen. (Compare Figures 1 and 2.)

Microscopically the tumor is seen to consist of fetal thymic tissue, that is, it shows epithelial cells in solid masses or arranged in a network among which there are varying numbers of small lymphocytes. The tissue corresponds to the fetal thymus at the stage when the epithelial organ is transformed into a lymphoid organ. No Hassall's corpuscles are present.

No lesions are found in the tissues of the scalp. The dura mater readily separates from the calvarium. The sinuses of the dura show no lesions. The subarachnoid contains a considerable amount of clear fluid in its dependent portions. The arteries of the brain are soft. The ven- 
tricles of the brain are not dilated and appear normai. A central coronal section through the brain shows no lesion.

The lumbar and thoracic part of the cervical spinal cord are removed. The meninges show no lesions. Sections of the cord show no gross lesions, except a possible softening in the anterior horns of the cervical cord.

The muscles examined were a few pieces from the sternothyroid and sternohyoid. These did not show lymphorrhages.

Of fifty-six necropsies on cases of myasthenia gravis that have been recorded since 1901, seventeen showed hyperplasia or persistence of thymus, and ten of the fifty-six cases showed a thymic tumor. This gives a thymic involvement in nearly half of the cases. All the thymic tumors except one have been of the benign type, somewhat similar to the case reported. The authorities seem to be very general in their statements that these tumors are special forms of hyperplasia, and not true neoplasms, and therefore can best be designated as benign thymomas.

The literature on the thymus gland is neither convincing nor satisfying. The fact seems to be generally accepted that the thymus and the thyroid are interrelated, particularly from a circulatory point of view. The assumption is quite clear, too, that the thyroid is responsible for disturbances of bodily metabolism; and it seems reasonably safe to assume that the thymus is in some way responsible for like conditions. Some investigators believe that the thymus and the lymphatic system are coordinated in function, and that the thymus itself is probably concerned in the production of lymphocytes. It is further probable that the thymus has some correlated effect with other ductless glands; and, like other glands, the thymus may be the primary seat of the attack. This does not necessarily mean that the thymus itself is an independent organ, but that it has its influence in the ductless gland chemistry, and certain persons have disorders and hyperplasias or diseases of the thymus, while in others the same conditions cause disturbance of other glands, as the thyroid or the suprarenals. One can only theorize on the whole situation concerning the dependence of the thyroid, thymus, and suprarenals, one on the other. The condition which we look on as status lymphaticus is something that we know very little about. We are led to believe from various sources that this condition may cause death under certain surroundings or circumstances. The same applies to the thymus, that there may be a status thymaticus or, as has been more aptly expressed, a status thymicolymphaticus. Some investigators do make a definite distinction between status thymicus, status lymphaticus, and status thymicolymphaticus; but this distinction can be demonstrated only after death. The group of symptoms which occur before death is not very readily differentiated.

The presence of a thymus in a person of middle life, particularly when associated with an exophthalmic goiter, is a very strong index that the thymus is the main disturbing element; and not infrequently the operator who removes the thyroid gland without recognizing the presence of a thymus hyperplasia produces a change in the circulation of the thymus which, not infrequently, is accompanied by sudden death.
There is every reason to suppose, therefore, that sudden deaths in these cases of myasthenia gravis in which the enlarged thymus is present may occur, as in the case earlier recorded in this paper, and the death be a thymic death.

The relationship between thymic disease and the central nervous system has never been established, unless it be that certain types of people who have inherent tendencies or inherent defects may be subjects of hyperplasias of the thymus, as well as other organs; and this, in turn, may have produced a change in the blood stream of the brain stem, either from indirect pressure or from substances thrown into the circulation which affect the respiratory or circulatory centers. The majority of thymic deaths in young people and children are probably due to pressure effects, yet this does not eliminate the probability of toxins in the blood stream, as well as pressure from overgrowth. This theory is based on the experiments of a few of the observers who have found nerve lesions, particularly in the gray cells, in people who have thymoma.

\section{ABSTRACT OF DISCUSSION}

Dr. Walter B. Swift, Boston: It has been held by Brown of Brooklyn that thymus enlargement is an etiologic factor in stammering. I should like to ask Dr. Jones if any of the cases he knows about have stammering connected with this lesion.

Dr. R. Foster KenNedy, New York: In my first two years at the National Hospital I did not see any case of myasthenia gravis. Later we had nine cases inside of eighteen months. Seven of these patients died; we succeeded in getting six postmortem examinations. Of the six cases, five had thymic persistence. In practically all of these the thymus was as large as my hand and was as thin as tissue paper, comprising a thin sheet of glandular tissue which spread down over the whole of the upper part of the pericardium. While it was not clear to us a half dozen years or so ago what the connection between the clinical disease and these extraordinary pathologic findings might be, it did occur to our dull intelligence that it was more than a coincidence, that there must be here some relation between cause and effect. I have seen only one case of myasthenia gravis in a woman over 25 . They were nearly all young women; the men patients were always in middle life. Sir William, Gowers published a group of cases of three men as I remember, which he calls pseudomyasthenia gravis, as a result of gasoline poisoning, or as he called it, petrol poisoning.

Dr. C. Eugene Riggs, St. Paul: I have under my care at the present time a case of myasthenia gravis in a woman who is over 25 and under 30 .

Dr. George A. Moleen, Denver: A case came under my observation as a dysentery and in Colorado when we find dysenteries, emaciation and marked prostration one of the first assumptions is "tuberculosis." On inquiry it was learned that it was not lung trouble for which this patient came West, but a muscular weakness. He had been examined in Chicago by a very good man some twenty-four years before and the condition was then pronounced a progressive muscular atrophy ; six years later it was called myasthenia gravis. The myasthenia was evident, and it occurred in epochs. The acute attacks occurred in periods of two or two and one half years, with all the bulbar and ocular symptoms, then he would gradually recover, gaining in strength. But since the last two or three attacks prior to observation he had not recovered completely. Examination revealed tenderness under the right 
lower costal margin at that time, and very fortunately segregation of the urine revealed pus coming from the right kidney. Exploratory operation revealed a large perinephritic abscess which compressed the right suprarenal against the lower surface of the liver on that side. Evacuation and drainage of that caused prompt return of his power. I followed the case for about six months after that, when he returned to Kansas, and took up his occupation. The case was distinctly myasthenic in type, and at that time was reported as a case of Addison's disease from a perinephritic abscess.

Dr. William A. Jones, Minneapolis: In the literature on myasthenia gravis I found nothing relating to speech defects, particularly stammering. I have seen but few cases of what I believed were true myasthenia gravis, and the two or three I have in mind at the present time have manifested no marked change, and are still living fairly comfortable lives. The tumor in the case I reported was about the size of a large Damask plum, and naturally occupied a considerable space in the mediastinal region.

\section{EXPERIMENTAL STUDIES OF INJECTION OF THE GASSERIAN GANGLION}

$$
\begin{gathered}
\text { CONTROLLED BY FLUOROSCOPY * } \\
\text { LEWIS J. POLLOCK, M.D. } \\
\text { HOLLIS E. POTTER, M.D. } \\
\begin{array}{c}
\text { CHICAGo } \\
\text { ChD }
\end{array}
\end{gathered}
$$

A perusal of the contemporaneous literature leads to the conclusion that gasserian ganglion operations and intraneural or intraganglionic injections of alcohol are the procedures of selection for the treatment of trifacial neuralgia.

The technical difficulties of operations on the gasserian ganglion or its sensory root and the relatively high mortality attending such operations, except in the hands of a very few surgeons, made welcome a procedure which offered at least alleviation without danger, disagreeable sequelae or great difficulty.

The injection of pain deadening and nerve destroying substances into the trifacial nerve dates back to 1874, when Bartholow reported successes with the injection of chloroform. Successful results with the injection of osmic acid were recorded through a period of years following Billroth and Neuber in 1884, by Eulenberg, Shapiro, Bennet, Murphy, Wright and others.

It was not until Schlösser reported the results of his experiments in 1903 that persistent efforts were inspired for the development of a technic for successful alcoholization of the fifth nerve. About the same time further impetus was given by the work of Ostwald, Levy and Baudoin. The technic of the latter two was adopted by Patrick, Hecht, Sicard, Harris and others. The development of the technic and the reports of its successful employment by these workers has placed this procedure permanently among the methods employed for the effective relief of trifacial neuralgia.

It is not within the scope of this paper, which deals with a technical problem, to describe the details of the development of methods of injection and their results.

Suffice it to say that in the hands of operators who have had wide experience and who have developed consummate skill, all these methods are uncertain. As

* Read before the Section on Nervous and Mental Diseases at the Sixty-Seventh Annual Session of the American Medical Association, Detroit, June, 1916.
Patrick" says, "It requires little experience or reflection to realize that no one can uniformly touch with a needle a given point 4 to $5 \mathrm{~cm}$. (2 inches) below the surface."

The uncertainty of prevailing methods led us to pursue some experimental work on which this paper is based. We are concerned with reaching the gasserian ganglion through the foramen ovale. Part of the experiments deal with a route which Harris uses in his technic. He, with Patrick and others, employs with little modification the course adopted by Levy and Baudoin. The needle is inserted in the sigmoid notch from 1.5 to $2.5 \mathrm{~cm}$. anterior to the anterior root of the zygoma or from a point immediately in front of the preglenoid tubercle, to one a centimeter farther forward. The needle is directed somewhat backward and slightly forward, and at $4 \mathrm{~cm}$. should reach the nerve. The needle arrives at the foramen ovale after having passed through the skin, subcutaneous tissue, the zygomatic insertion of the masseter, posterior portion of the

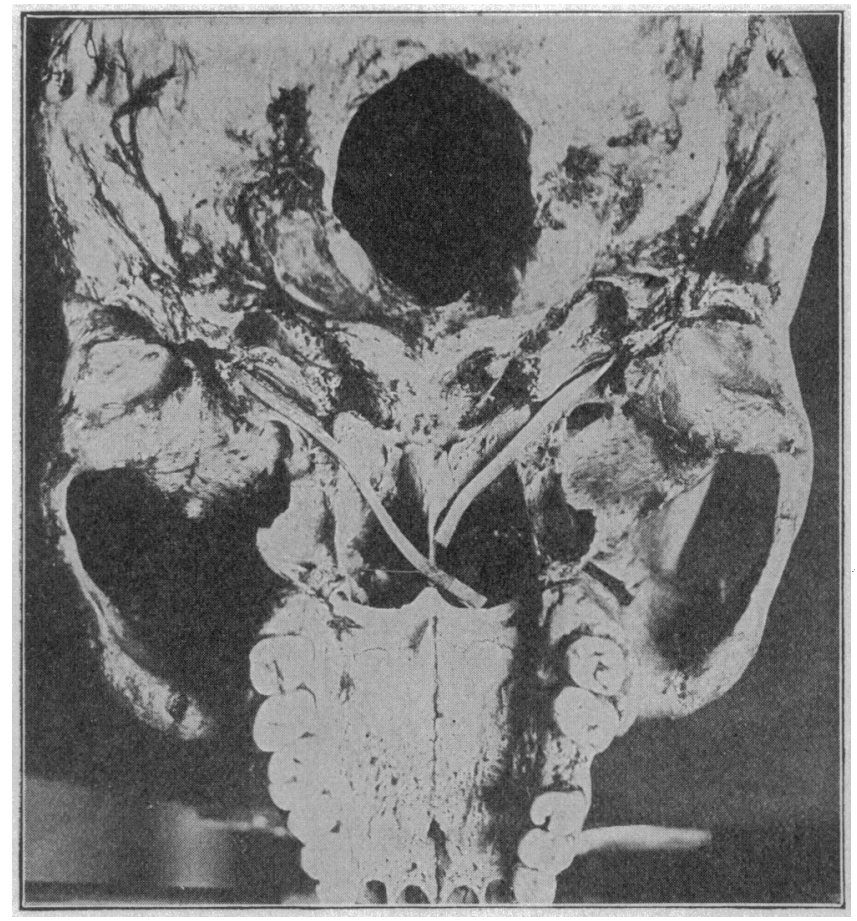

Fig. 1.-Lead strips in the groove of the eustachian tube, showing the foramen ovale immediately external and anterior to it.

temporal tendon, superior border of the externai pyterygoid muscle and lastly in the front of the temporo-maxillary articulation. Patrick ${ }^{2}$ has called attention to the fact that:

A really valuable landmark is the external pterygoid plate. The foramen ovale, at least in the vast majority of cases, is directly back of this and at about the same depth. Hence I aim to strike this plate close to its posterior edge and then work backward until the needle slips by it. Then I have every reason to believe that the point of the needle is just in front of the nerve and at about the proper depth.

Patrick gives a very valuable guide to be used in determining the approximate depth of the foramen from the surface. Analysis of his measurements in

1. Patrick, Hugh T.: The Treatment of Trifacial Neuralgia by Means of Deep Injections of Alcohol, THE Journal A. M. A., Nov. 9, 1907 , p. 1567.

2. Patrick, Hugh T.: The Technic and Results of Deep Injections of Alcohol for Trifacial Neuralgia, The Journal. A. M. A., Jan. 20, 1912 , p. 155 . 